Article

\title{
Analysis of the Graovac-Pisanski Index of Some Polyhedral Graphs Based on Their Symmetry Group
}

\author{
Modjtaba Ghorbani ${ }^{1, * \mathbb{C}}$, Mardjan Hakimi-Nezhaad ${ }^{1} \mathbb{D}$, Matthias Dehmer ${ }^{2,3,4}{ }^{(}$and Xueliang Li ${ }^{5}$ \\ 1 Department of Mathematics, Faculty of Science, Shahid Rajaee Teacher Training University, \\ Tehran 16785-136, Iran; m.hakiminezhaad@sru.ac.ir \\ 2 Department of Computer Science, Swiss Distance University of Applied Sciences, 3900 Brig, Switzerland; \\ Matthias.Dehmer@umit.at \\ 3 Department of Biomedical Computer Science and Mechatronics, UMIT, A-6060 Hall in Tyrol, Austria \\ 4 College of Artficial Intelligence, Nankai University, Tianjin 300071, China \\ 5 Center for Combinatorics, Nankai University, Tianjin 300071, China; lxl@nankai.edu.cn \\ * Correspondence: mghorbani@sru.ac.ir; Tel.: +98-21-2297-0029
}

Received: 6 July 2020; Accepted: 7 August 2020; Published: 25 August 2020

\begin{abstract}
The Graovac-Pisanski (GP) index of a graph is a modified version of the Wiener index based on the distance between each vertex $x$ and its image $\alpha(x)$, where $\alpha$ is an automorphism of graph. The aim of this paper is to compute the automorphism group of some classes of cubic polyhedral graphs and then we determine their Wiener index. In addition, we investigate the GP-index of these classes of graphs.
\end{abstract}

Keywords: automorphism group; polyhedral graph; graph invariant

\section{Introduction}

In the current work, all graphs are finite, undirected and connected. Two symbols $V(G)$ and $E(G)$ show the vertex and edge sets of $G$, respectively. If $x$ and $y$ are two given vertices of a graph $G$, then the distance between them, denoted by $d(x, y)$, is the length of each shortest path connecting them.

Let $u v \in E(G)$ be an edge of a graph $G$. A permutation $\alpha$ on the vertex set $V(G)$ is an automorphism of $G$, if $u v \in E(G)$ if and only if $\alpha(u) \alpha(v) \in E(G)$, where $\alpha(u)$ is the image of vertex $u$. Hence, an automorphism group is a set of all automorphisms of $G$ and we denote it by $A u t(G)$.

For any vertex $u \in V(G)$ an orbit of $G$ containing $u$ is defined as $u^{G}=\mathcal{O}(u)=\{\alpha(u): \alpha \in A u t(G)\}$. We say $G$ is vertex-transitive if it has only one orbit. Equivalently, a graph is vertex-transitive if for two vertices $u, v \in V(G)$ there is an automorphism $\sigma \in A u t(G)$ such that $\sigma(u)=v$.

The Wiener number of a graph is a distance-based graph invariant defined as the sum of distances between all pairs of vertices in $G$. In other words,

$$
W(G)=\frac{1}{2} \sum_{u, v \in V(G)} d(u, v) .
$$

The Wiener index is a remarkable predictor (among all graph descriptors) for predicting the boiling point of some molecules such as alkanes and paraffin, see [1]. This quantity is considered by the scientists at the beginning of the development of QSPR/QSAR approaches to chemistry-related problems. It is defined as the half sum of the distances between all pairs of vertices in a graph. Motivated by this single detail, several mathematically oriented investigations of Wiener index were undertaken in [2-4] as well as [5-7]. 
Suppose $G$ is a graph with automorphism group $A=A u t(G)$. Then the Graovac-Pisanski index is defined as [8]

$$
\widehat{W}(G)=\frac{|V(G)|}{2|A|} \sum_{x \in V(G)} \sum_{\alpha \in A} d(x, \alpha(x)) .
$$

Črepnjak et al. showed that the GP-index of some hydrocarbon molecules is correlated with their melting points [9]. We refer to [10] for mathematical properties and chemical meaning of this graph invariant.

Ashrafi et al. [11] computed the GP-index of some graph products and in [12], some upper and lower bounds for this graph invariant are presented. In 2016, Ghorbani and Klavžar [13] computed this graph invariant by a cut method and Tratnik [14], by generalizing their method, achieved closed formulas for the GP-index of zig-zag tubulenes.

Recently, some papers are devoted to finding the extremal graphs with respect to GP number, see [15,16]. In [17] it is proved that if $T$ is a tree, then $\widehat{W}(T) \leq W(T)$ and in [18] the authors showed if $G$ is either a connected bipartite graph or a connected graph of even order, then $\widehat{W}(G)$ is an integer. This difference between the Wiener index and GP-index was first considered in [19], and in [20] this quantity was computed for some families of polyhedral graphs. Knor et al. [21] considered the class of trees and they proved that this value is non-negative.

For more details about the GP number of nanostructures, linear polymers, some classes of fullerenes and fullerene-like molecules, see $[8,10-14,19,20,22-31]$. However, the following result is crucial in the whole of this paper.

Theorem 1. [32] (The orbit-stabilizer property) Let $G$ acts on the set $X$ and $x \in X$. If $G$ is finite, then $|\mathcal{O}(x)| \times\left|G_{x}\right|=|G|$, where $G_{x}=\{g \in G: g(x)=x\}$.

\section{Main Results}

A polyhedral graph is a three connected planar graph and a fullerene is a cubic polyhedral graph, see [33-35].

The Wiener and GP-indices of several infinite classes of fullerene and polyhedral graphs have been computed in $[19,20,23]$ as well as [24-26]. The aim of this paper is to explore these quantities for some new classes of graphs. In [36] a method is described to obtain a fullerene graph from a zig-zag or armchair nanotubes.

Consider an infinite class of fullerene graph with pentagons and hexagons as depicted in Figure 1. This class of fullerene graphs has exactly $20 n+4$ vertices, where $n \geq 3$.
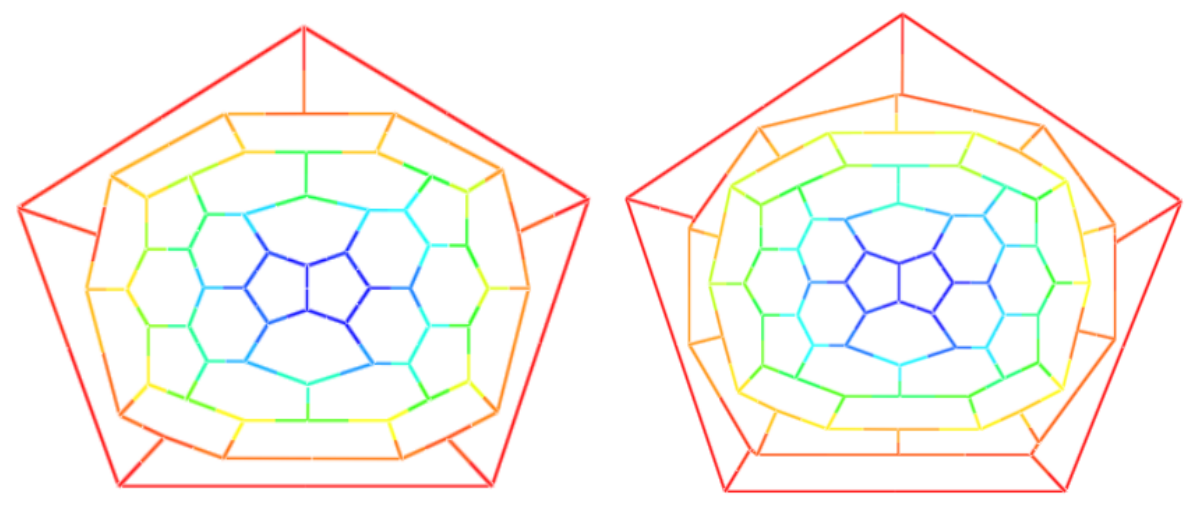

Figure 1. Fullerene $F_{20 n+4}$, where $n=3$ (left) and $n=4$ (right).

We show a nanotube made up of $m$ rows and $n$ columns of hexagons by $T_{z}[m, n]$, see Figure 2 . As it is shown in Figure 3, combining a nanotube $T_{z}[10, n]$ from both sides by two caps $B$ and $C$ 
(Figure 4) results in a fullerene graph on $20 n+4$ vertices, $n \geq 3$. In this way, we can construct many classes of polyhedral graphs such as fullerenes. In continuing this section, we introduce some infinite families of cubic polyhedral graphs.

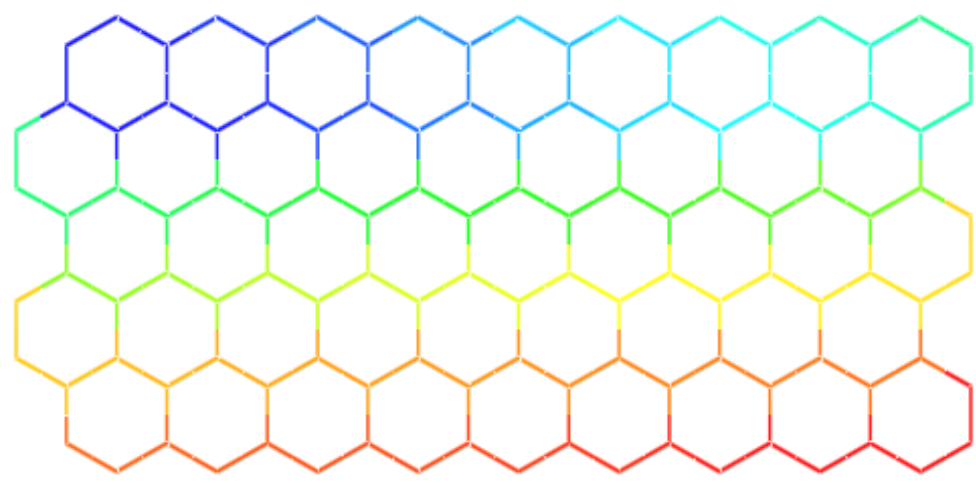

Figure 2. Two-dimensional graph of zig-zag nanotube $T_{z}[m, n]$, for $m=10, n=5$.
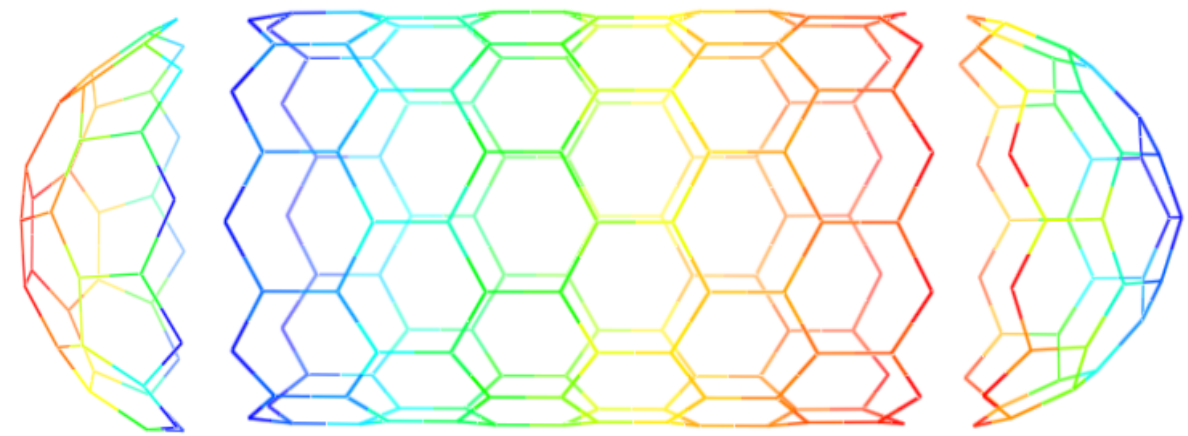

Figure 3. Fullerene $F_{20 n+4}$ created by combining a copy of cap $B$, a copy of a copy of cap $C$ and a copy of zig-zag nanotube $T_{z}[10, n]$.
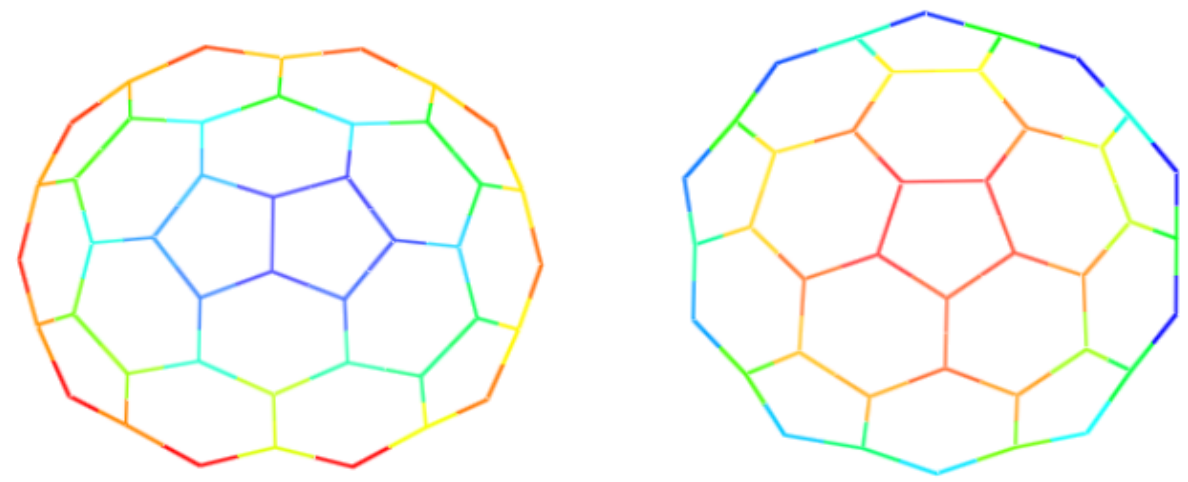

Figure 4. Two caps $B$ (left) and $C$ (right).

\section{1. (4,5,6)-Cubic Polyhedral Graphs}

At first, we demonstrate an infinite class of cubic polyhedral graphs composed of quadrangular, pentagonal and hexagonal faces, see Figure 5 . It has exactly $12 n+10$ vertices where $n \geq 3$ and hence we denote it by $F_{12 n+10}$. That is a $(4,5,6)$-cubic polyhedral graph. 

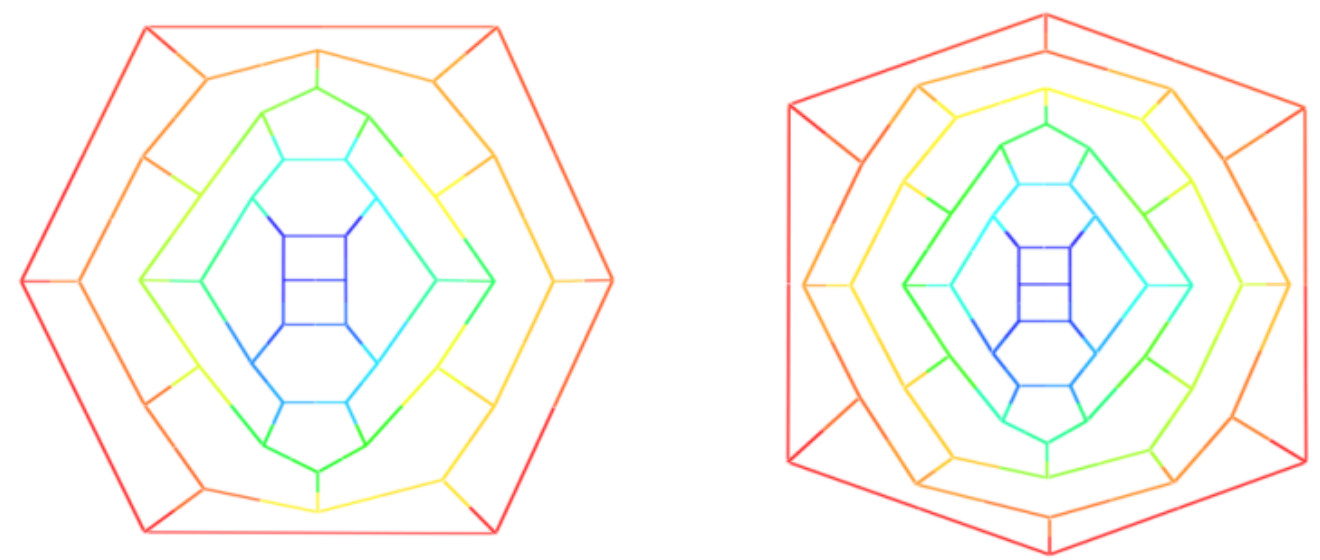

Figure 5. The polyhedral graph $F_{12 n+10}$, where $n=3$ (left) and $n=4$ (right).

To construct this class of graphs, combine a nanotube $T_{Z}[6, n]$ (Figure 2) with a copy of a copy of cap $B$, a copy of cap $C$ (Figure 6) as shown in Figure 7 to form a polyhedral graph with $12 n+10$ vertices, where $n \geq 3$.
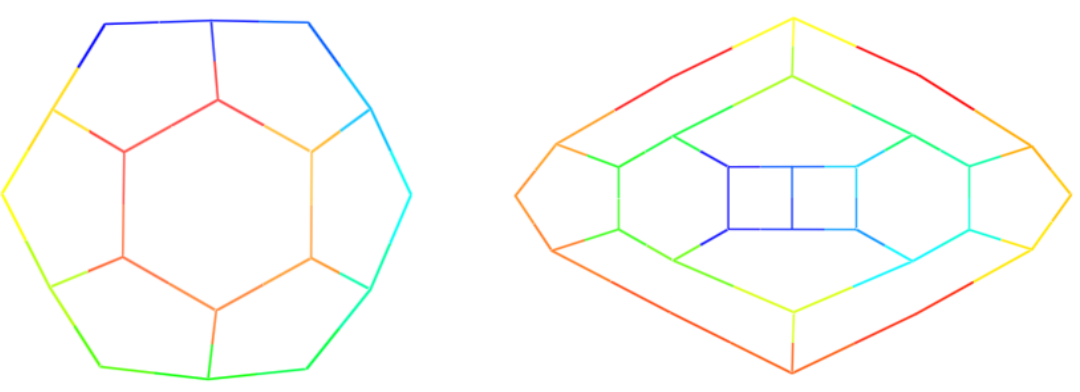

Figure 6. Two caps $B$ (left) and $C$ (right).
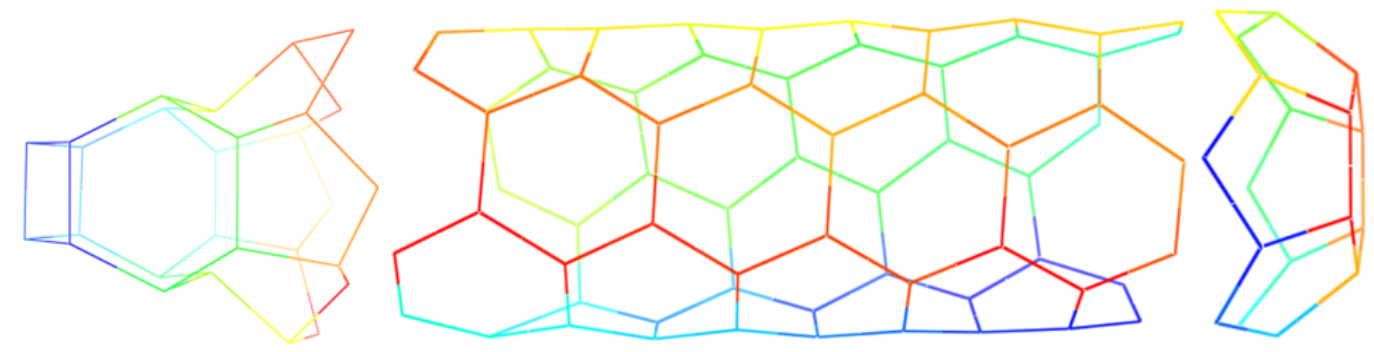

Figure 7. Polyhedral graph $F_{12 n+10}$ constructed by combining a copy of cap $B$, a copy of a copy of cap $C$ and a copy of zig-zag nanotube $T_{Z}[6, n]$.

\section{2. (4,6,7,8)-Cubic Polyhedral Graphs}

A cubic polyhedral graph containing squares, hexagons, heptagons and octagons is denoted by a $(4,6,7,8)$-polyhedral graph, see Figure 8. If we combine a nanotube $N_{Z}[8, n]$ (Figure 9) with a copy of cap $B$ and a copy of cap $C$ (Figure 10) as shown in Figure 11, the resulted graph is denoted by $F_{24 n}$, which has $24 n$ vertices. 

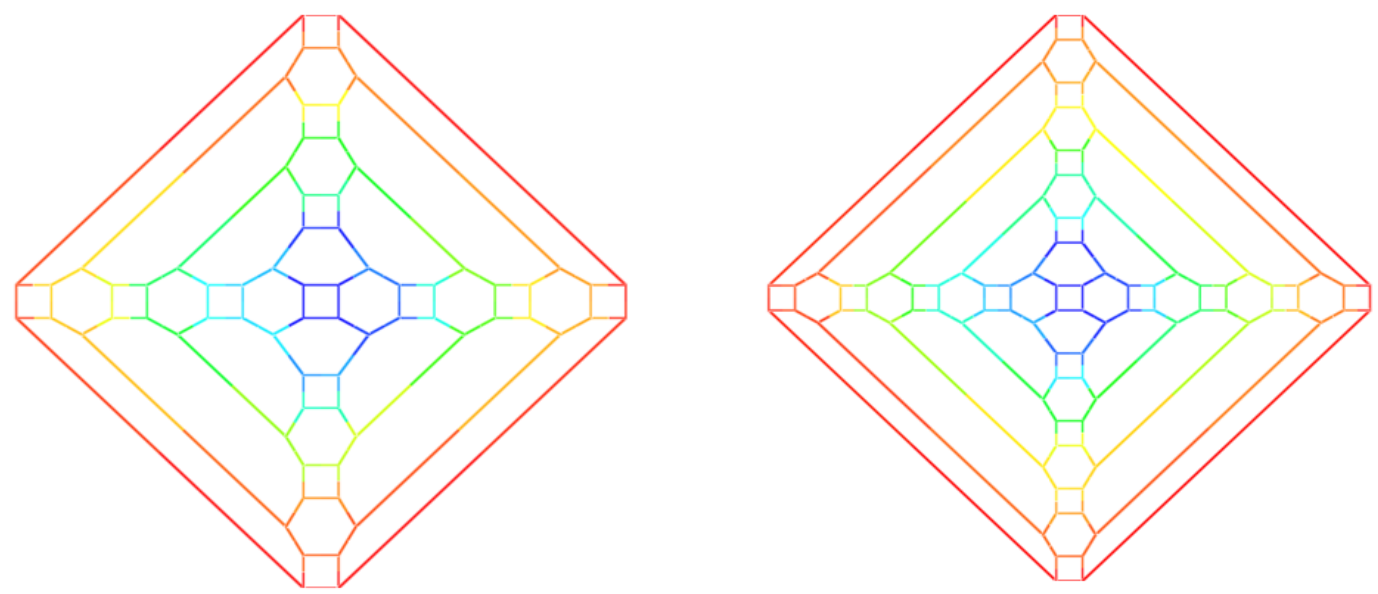

Figure 8. Two-dimensional graph of $F_{24 n}$ for $n=3$ (left) and $n=4$ (right).

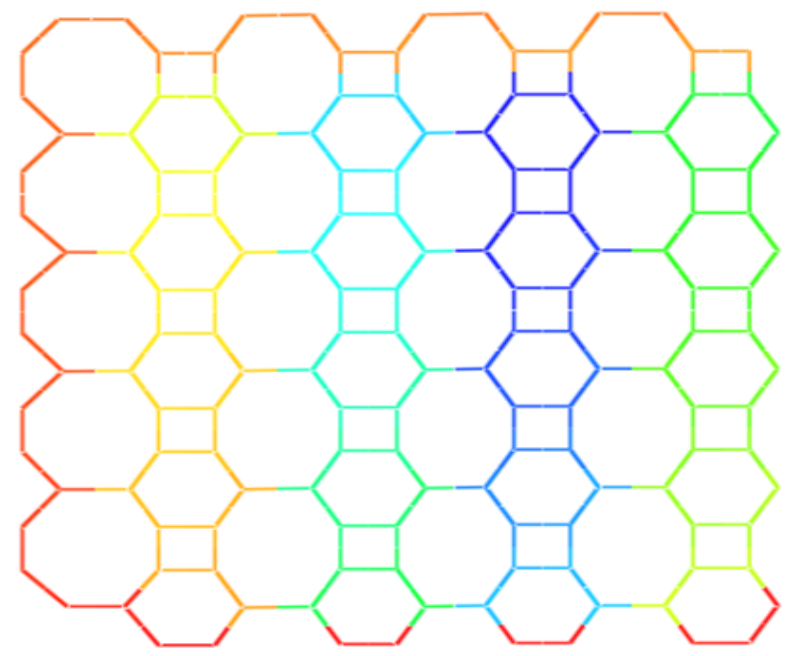

Figure 9. Two-dimensional graph of nanotube $N_{Z}[m, n]$, for $m=8, n=5$.
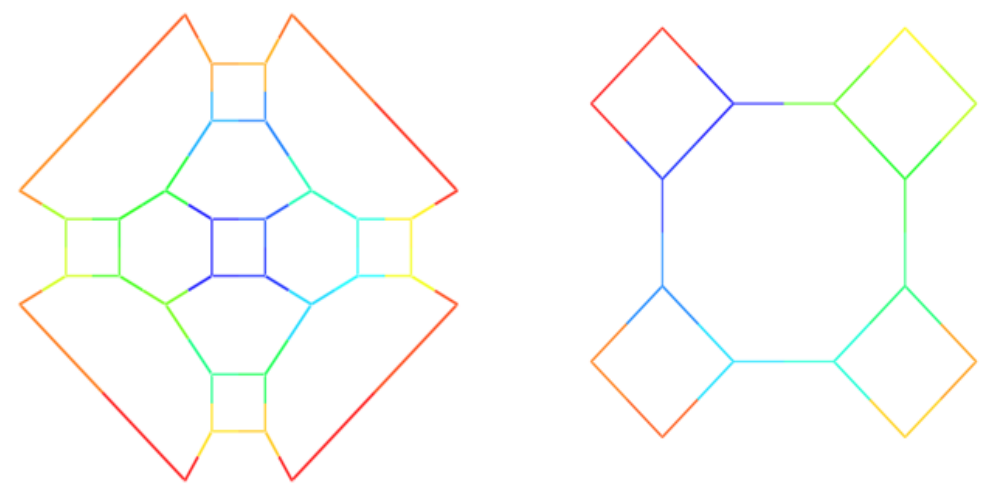

Figure 10. Two caps $B$ (left) and $C$ (right). 

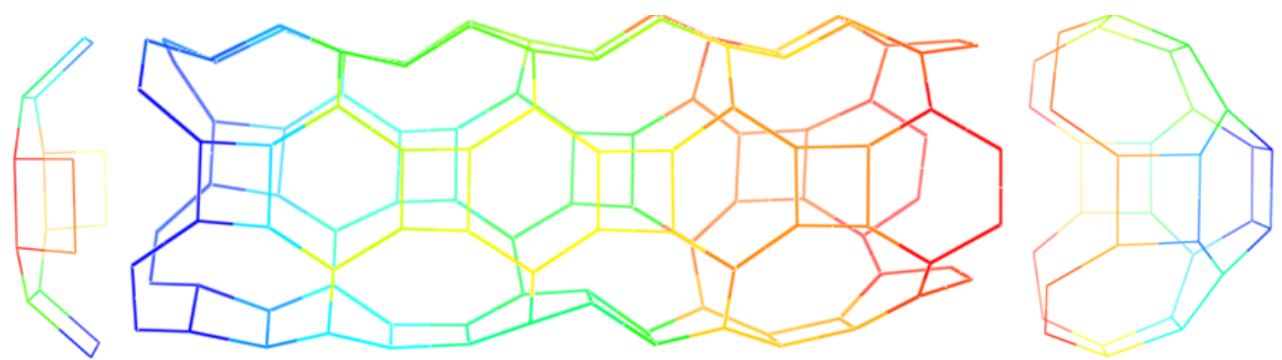

Figure 11. Polyhedral graph $F_{24 n}$ made up by combining a copy of cap $B$, a copy of cap $C$ and a copy of $N_{z}[8, n]$.

\section{3. $(4,6,8)$-Cubic Polyhedral Graph}

In continuing, we introduce two classes of cubic polyhedral graphs containing the quadrilateral, the hexagonal and the octagonal faces. At first, consider the polyhedral graph $F_{16 n}$ in Figure 12. This graph has $16 n(n \geq 3)$ which can be constructed in a similar way to the last ones, see Figures 13 and 14.
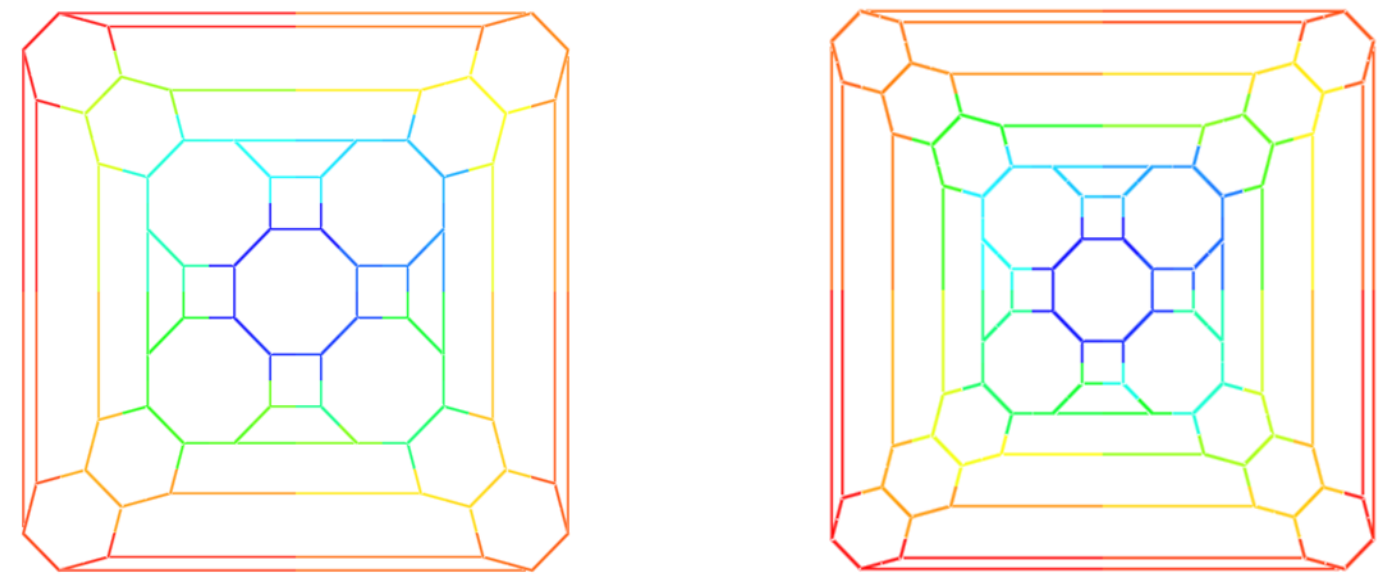

Figure 12. The polyhedral graph $F_{16 n}$, where $n=4$ (left) and $n=5$ (right).
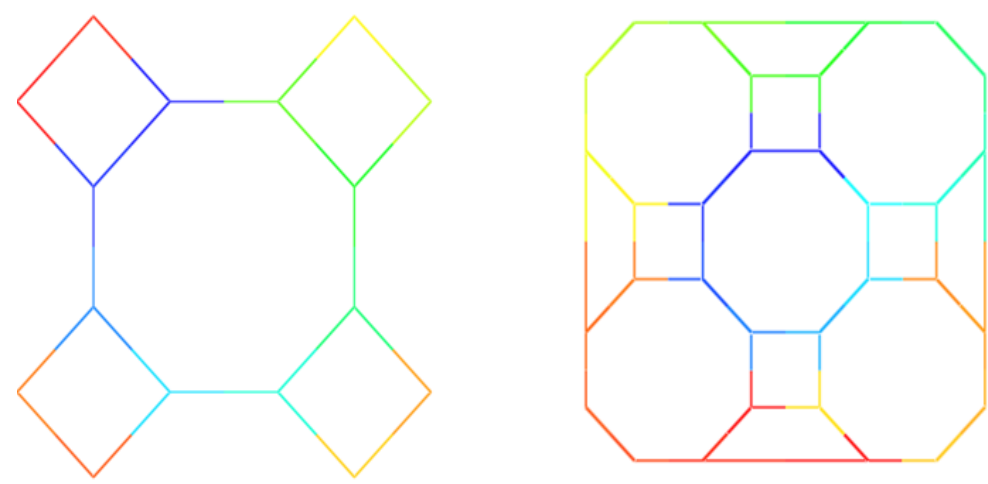

Figure 13. Two caps $B$ (left) and $C$ (right). 


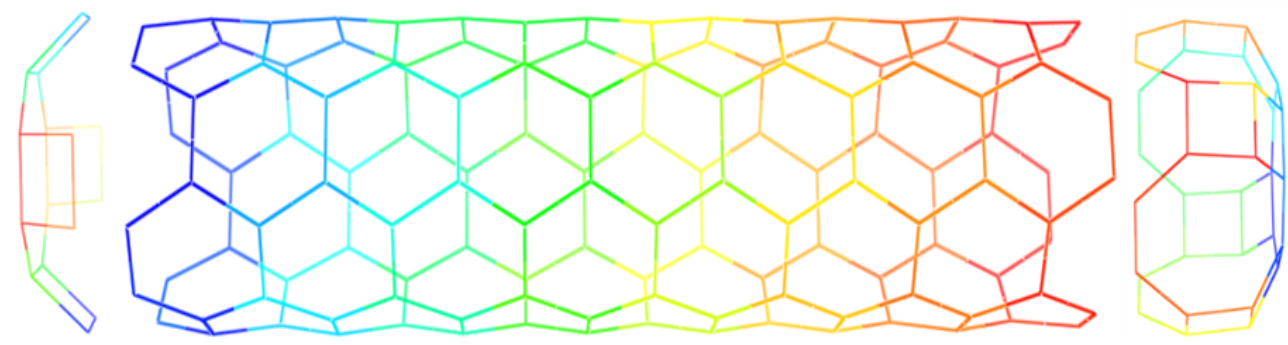

Figure 14. Polyhedral graph $F_{16 n}$ created by combining a copy of cap $B$, a copy of a copy of cap $C$ and a copy of zig-zag nanotube $T_{Z}[8, n]$.

Another class of polyhedral graphs, denoted by $F_{16 n}$, is depicted in Figure 15 which has $24 n+16$ vertices $(n \geq 3)$. In Figures 16 and 17, the construction of $F_{24 n+16}$ is depicted.

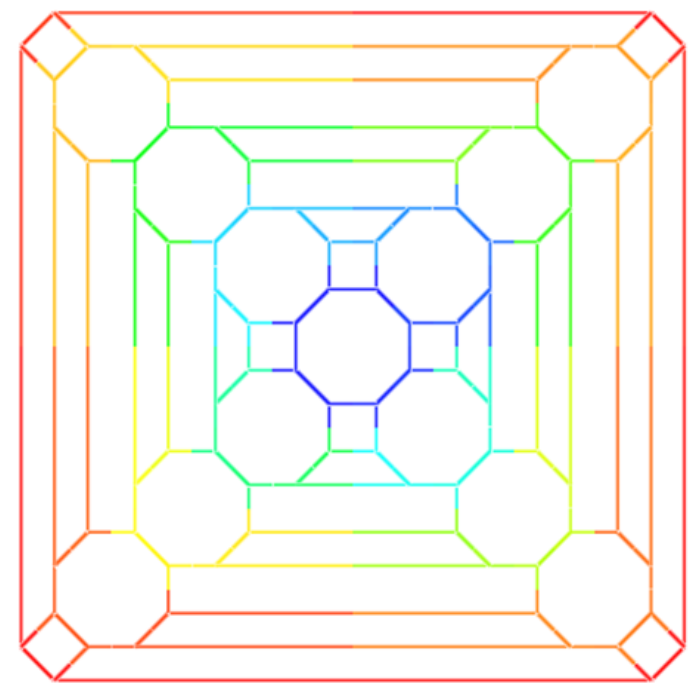

Figure 15. The polyhedral graph $F_{24 n+16}$ for $n=3$.
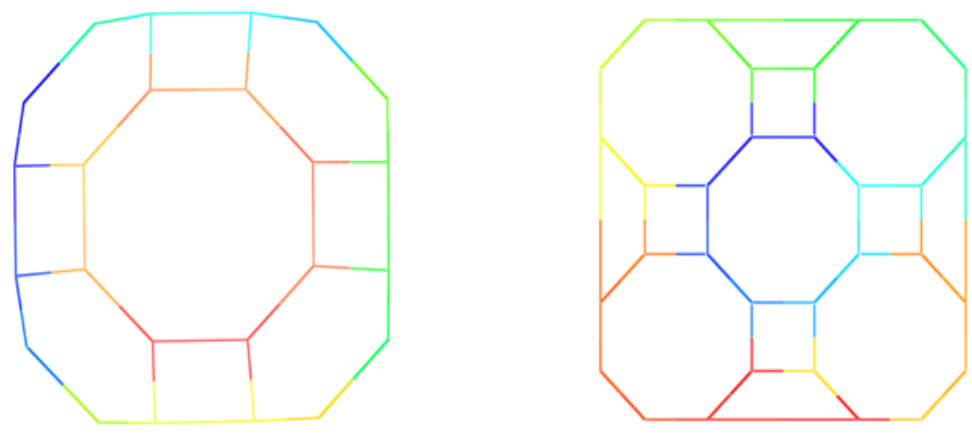

Figure 16. Two caps $B$ (left) and $C$ (right). 

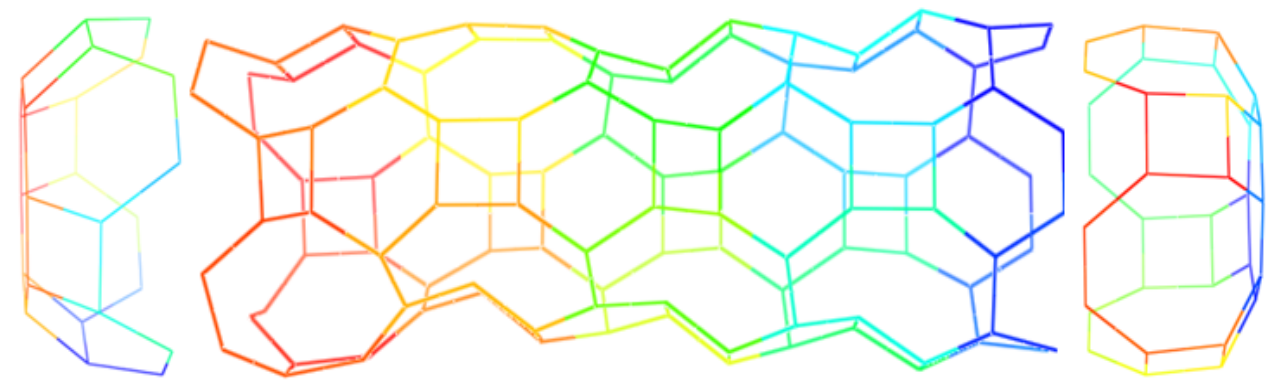

Figure 17. Polyhedral graph $F_{24 n+16}$ made up by combining a copy of cap $B$, a copy of cap $C$ and a copy of $N_{z}[8, n]$.

\section{Symmetry Group of Polyhedral Graphs}

The aim of this section is to compute the automorphism group of polyhedral graphs introduced in this paper. We explain our methods only for small ones and in a similar way, we can determine the automorphism group of each member of related class. For more details on the automorphism group of polyhedral graphs, see [37-39].

In continuing this section, two symbols $\mathbb{Z}_{2}$ and $D_{8}$ are defined to show a cyclic group with two elements and a dihedral group with eight elements, respectively. The dihedral group arises as the symmetry group of many classes of molecular graphs. This group has $2 n$ elements and a presentation of it is as follows:

$$
D_{2 n}=\left\langle\alpha, \beta \mid \alpha^{n}=\beta^{2}=1, \beta \alpha \beta=\alpha^{-1}\right\rangle \text {. }
$$

Theorem 2. The automorphism group $A=A u t\left(F_{16 n}\right)$ is isomorphic with dihedral group $D_{8}$.

Proof. For $n=3$, a labeling of vertices of $F_{16 n}$ is depicted in Figure 18. Suppose $\alpha$ indicates a rotation element of the $F_{16 n}$ through an angle of $45^{\circ}$. Then we can prove that $1^{\langle\alpha\rangle}=\{1,3,5,7\}$, where $\langle\alpha\rangle$ is a subgroup generated by $\alpha$. Now, consider the axis symmetry $\beta$ which fixes no vertices, we have, $\mathrm{A} \geq\langle\alpha, \beta\rangle$. By the orbit-stabilizer property, we obtain $|A|=\left|2^{A}\right| \times\left|A_{2}\right|$. Since no element fixes 2, we have $\left|A_{2}\right|=1$ or equivalently $|A|=\left|2^{A}\right|$ and thus $\left|2^{\langle\alpha, \beta\rangle}\right|=8$. By applying the method of [13] one can verify that $A=\langle\alpha, \beta\rangle \cong D_{8}$ and the proof is complete.

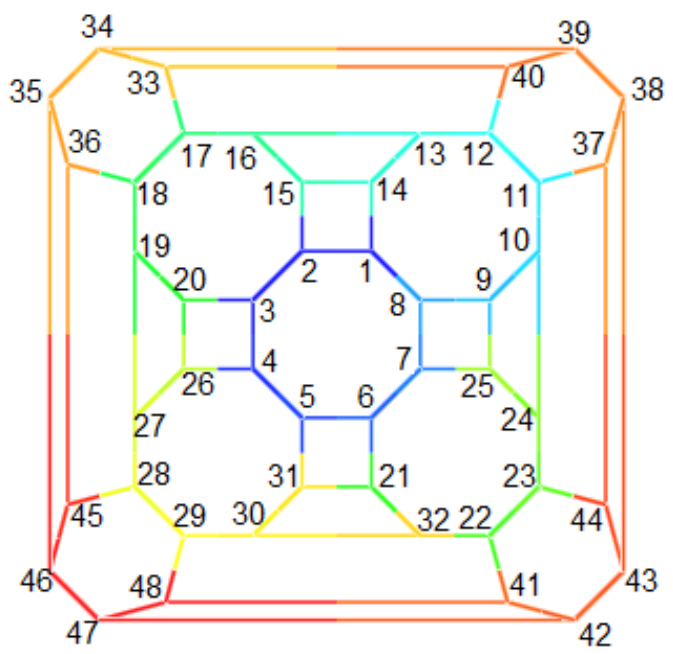

Figure 18. Labeling of cubic polyhedral graph $F_{16 n}$, where $n=3$. 
Theorem 3. The automorphism group of $F_{24 n+16}$ is isomorphic with dihedral group $D_{8}$.

Proof. Similar to the last cases, we compute the structure of $A=\operatorname{Aut}\left(F_{24 n+16}\right)$, where $n=2$, see Figure 19. If $\alpha$ denotes a rotation through an angle of $90^{\circ}$, then we have

$$
\begin{aligned}
\alpha= & (1,3,5,7)(2,4,6,8)(9,15,27,21)(10,16,28,22)(11,17,29,23)(12,18,30,24) \\
& (13,19,31,25)(14,20,32,26)(33,51,45,39)(34,52,46,40)(35,53,47,41) \\
& (36,54,48,42)(37,55,49,43)(38,56,50,44)(57,63,61,59)(58,64,62,60)
\end{aligned}
$$

and so $1^{\langle\alpha\rangle}=\{1,3,5,7\}$. Now, suppose

$$
\begin{aligned}
\beta= & (1,2)(3,8)(4,7)(5,6)(9,20)(10,19)(11,18)(12,17)(13,16)(14,15)(21,32)(22,31) \\
& (23,30)(24,29)(25,28)(26,27)(33,44)(34,43)(35,42)(36,41)(37,40)(38,39) \\
& (45,56)(46,55)(47,54)(48,53)(49,52)(50,51)(57,60)(58,59)(61,64)(62,63) .
\end{aligned}
$$

Then $A \geq\langle\alpha, \beta\rangle$ and orbit-stabilizer property yields that $|A|=\left|2^{A}\right|$, since $\left|A_{2}\right|=1$. But no automorphism such that $\sigma(u)=2$ and thus $2^{A}=\{1,2,3,4,5,6,7,8\}$. On the other hand $|\langle\alpha, \beta\rangle|=8$ where $\alpha^{4}=\beta^{2}=1, \beta \alpha \beta=\alpha^{-1}$ and then $A=\langle\alpha, \beta\rangle \cong D_{8}$. This completes the proof.

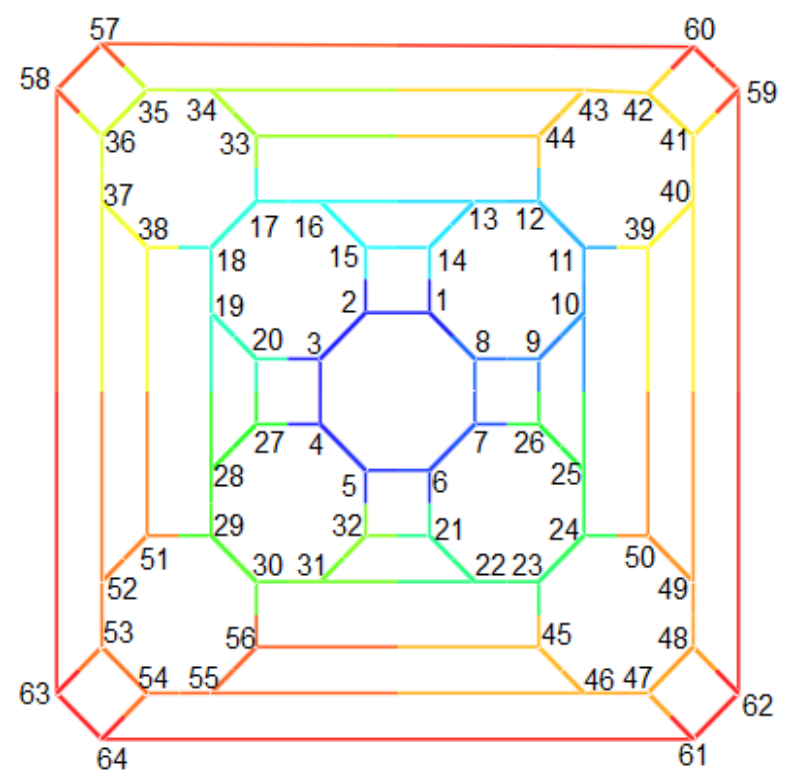

Figure 19. Labeling of cubic polyhedral graph $F_{24 n+16}$ for $n=2$.

Theorem 4. The automorphism group of $F_{20 n+4}$ is isomorphic with the cyclic group $\mathbb{Z}_{2}$.

Proof. Suppose $A=A u t\left(F_{20 n+4}\right)$ and $n=3$ (Figure 20). Suppose

$$
\begin{aligned}
\alpha= & (3,8)(4,7)(5,6)(9,12)(10,11)(13,14)(15,23)(16,22)(17,21)(18,20)(26,34)(27,33) \\
& (28,32)(29,31)(35,42)(36,41)(37,40)(38,39)(43,44)(45,50)(46,49)(47,48)(51,54) \\
& (52,53)(56,59)(57,58)(60,62)(63,64),
\end{aligned}
$$

then $|\langle\alpha\rangle|=2$, where $\alpha^{2}=1$. Hence, by the above discussion, the automorphism group can be computed as $A=\langle\alpha\rangle \cong \mathbb{Z}_{2}$. 


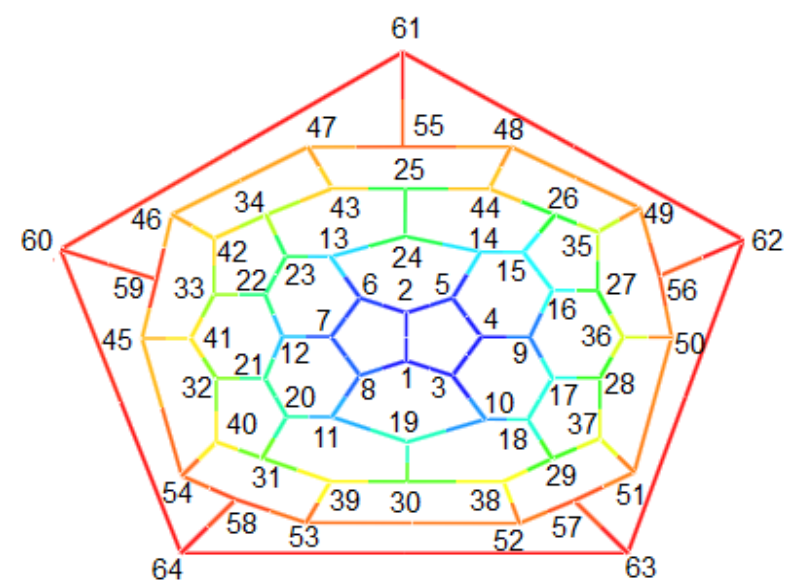

Figure 20. Labeling of fullerene graph $F_{20 n+4}$ for $n=3$.

Theorem 5. Aut $\left(F_{12 n+10}\right) \cong \mathbb{Z}_{2} \times \mathbb{Z}_{2}$.

Proof. Suppose $A=A u t\left(F_{12 n+10}\right)$ and $n=3$, as depicted in Figure 21. Similar to the proof of the last theorem, if $\alpha$ denotes the rotation of $F$ through an angle of $90^{\circ}$

$$
\begin{aligned}
\alpha= & (1,2)(3,4)(5,6)(7,10)(8,9)(11,14)(12,13)(15,16)(17,24)(18,20)(21,23)(25,28) \\
& (26,27)(30,40)(31,39)(32,38)(33,37)(34,36)(41,44)(42,43)(45,46),
\end{aligned}
$$

then $1^{\langle\alpha\rangle}=\{1,2\}$. Now, suppose

$$
\begin{aligned}
\beta= & (1,6)(2,5)(7,14)(8,13)(9,12)(10,11)(18,23)(19,22)(20,21)(25,26)(27,28)(29,35) \\
& (30,34)(31,33)(36,40)(37,39)(42,46)(43,45) .
\end{aligned}
$$

One can show that $|\langle\alpha, \beta\rangle|=4$, where $\alpha^{2}=\beta^{2}=1$ and $\beta \alpha \beta=\alpha^{-1}$. On the other hand, $A=\langle\alpha, \beta\rangle \cong D_{4} \cong \mathbb{Z}_{2} \times \mathbb{Z}_{2}$ and the proof is complete.

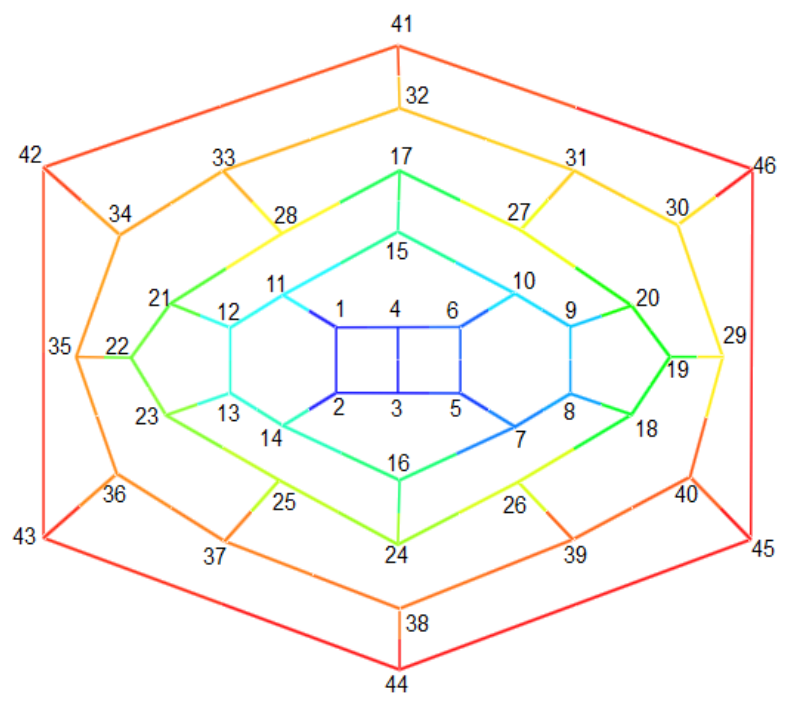

Figure 21. Labeling of vertices of polyhedral graph $F_{12 n+10}$ for $n=3$. 
Theorem 6. The automorphism group of $F_{24 n}$ is isomorphic with $D_{8}$.

Proof. Consider $F_{24 n}$, where $n=3$, as depicted in Figure 22, and suppose

$$
\begin{aligned}
\alpha= & (1,4,3,2)(5,8,11,14)(6,9,12,15)(7,16,13,10)(17,23,19,21)(18,24,20,22) \\
& (25,29,33,37)(26,30,34,38)(27,31,35,39)(28,32,36,40)(41,43,45,55)(42,44,46,56) \\
& (47,57,51,61)(48,58,52,62)(49,59,53,63)(50,60,54,64)(65,69,67,71)(66,70,68,72),
\end{aligned}
$$

and

$$
\begin{aligned}
\beta= & (1,3)(5,9)(6,8)(7,13)(11,15)(12,14)(17,22)(18,21)(19,24)(20,23)(25,40)(26,39) \\
& (27,38)(28,37)(29,36)(30,35)(31,34)(32,33)(41,56)(42,55)(43,46)(44,45)(47,64) \\
& (48,63)(49,62)(50,61)(51,60)(52,59)(53,58)(54,57)(65,72)(66,71)(67,70)(68,69) .
\end{aligned}
$$

It can be proved that $|\langle\alpha, \beta\rangle|=8$, where $\alpha^{4}=\beta^{2}=1, \beta \alpha \beta=\alpha^{-1}$ and hence $A=\langle\alpha, \beta\rangle \cong D_{8}$.

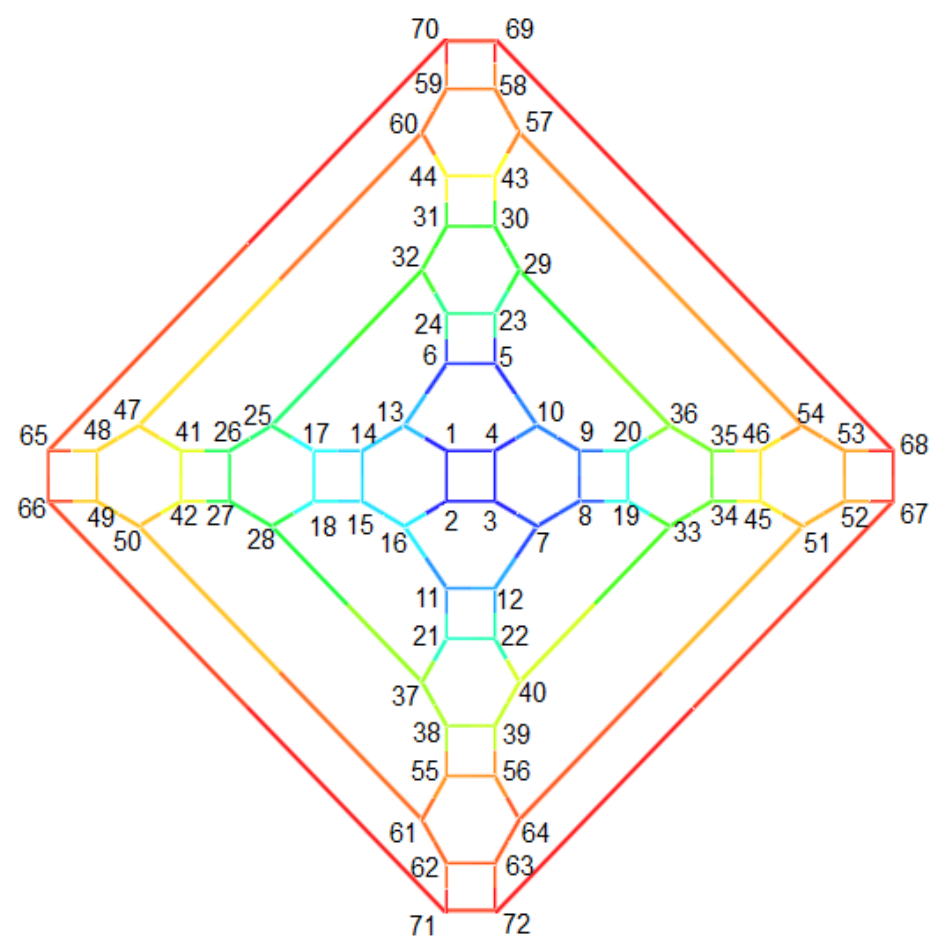

Figure 22. Labeling of cubic polyhedral graph $F_{24 n}$ for $n=3$.

\section{The Wiener Index and GP-Index of Polyhedral Graphs}

In this section, we investigate two descriptors introduced in this paper which are based on distances between vertices of a graph.

Theorem 7. Suppose $T=T_{Z}[6, n]$. For $n \geq 5$ we have

$$
W(T)=48 n^{3}+144 n^{2}+972 n-384 .
$$


Proof. The nanotube $T_{Z}[6, n]$ has exactly $n+1$ rows. Let us to show the vertices of the last row by $U=\left\{u_{1}, u_{2}, \ldots, u_{12}\right\}$. Let $t_{n}=2 W\left(T_{Z}[6, n]\right)$. A straightforward (but somehow lengthy) computation yields the recurrence

$$
\begin{aligned}
t_{n} & =\sum_{x, y \in U} d(x, y)+\sum_{x, y \in V \backslash U} d(x, y)+2 \sum_{x \in U, y \in V \backslash U} d(x, y) \\
& =432+t_{n-1}+2 \sum_{x \in U, y \in V \backslash U} d(x, y) .
\end{aligned}
$$

It is not difficult to see that

$$
\sum_{x \in U, y \in V \backslash U} d(x, y)=6\left(d\left(u_{1}\right)+d\left(u_{2}\right)\right),
$$

where $d\left(u_{1}\right)=\sum_{y \in V \backslash U} d\left(u_{1}, y\right)$ and $d\left(u_{2}\right)$ can be defined similarly, see Figure 23. A direct computation yields that $d\left(u_{1}\right)=12 n^{2}+18 n+40$ and $d\left(u_{2}\right)=12 n^{2}+6 n+70$. This implies that $t_{n+1}=432+t_{n}+$ $6\left(d\left(u_{1}\right)+d\left(u_{2}\right)\right)$. The solution of this recurrence is

$$
W(T)=48 n^{3}+144 n^{2}+972 n-384
$$

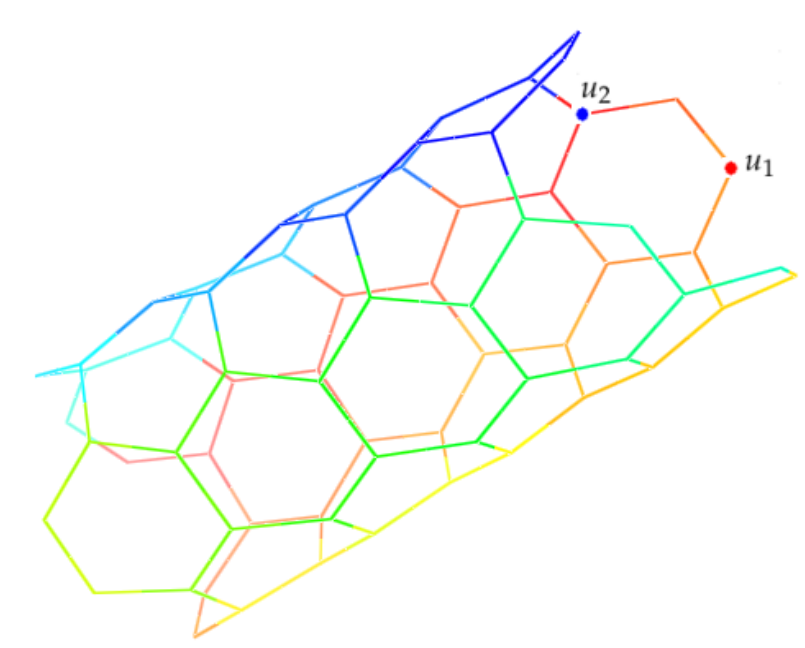

Figure 23. Three-dimensional graph of nanotube $T_{Z}[6, n]$.

Theorem 8. For the polyhedral graph $F=F_{12 n+10}$, for $n \geq 6$, we have

$$
W(F)=48 n^{3}+120 n^{2}+984 n-1086
$$

Proof. First, we determine the distance matrix of polyhedral graph $F$ which is a block matrix

$$
D=\left[\begin{array}{lll}
V & B & W \\
B & U & B \\
W & B & V
\end{array}\right]
$$

where $V, B$ and $W$ are distances between vertices of the first cap, $T_{Z}[6, n]$ and the second cap and where $\left\{v_{1}, v_{2}, \ldots, v_{r}\right\},\left\{u_{1}, u_{2}, \ldots, u_{s}\right\}$ and $\left\{w_{1}, w_{2}, \ldots, w_{r}\right\}$ are the set of vertices of the first cap, vertices of 
$T_{Z}[6, n]$ and vertices of the second cap, respectively. The matrix $U$ is the distance matrix of vertices $\left\{u_{1}, u_{2}, \ldots, u_{s}\right\}$. Then

$$
V=\left[\begin{array}{llllllllllllllllll}
0 & 1 & 2 & 3 & 4 & 5 & 6 & 5 & 4 & 3 & 2 & 1 & 3 & 4 & 4 & 3 & 2 & 2 \\
1 & 0 & 1 & 2 & 3 & 4 & 5 & 5 & 5 & 4 & 3 & 2 & 2 & 3 & 4 & 3 & 2 & 1 \\
2 & 1 & 0 & 1 & 2 & 3 & 4 & 5 & 6 & 5 & 4 & 3 & 2 & 3 & 4 & 4 & 3 & 2 \\
3 & 2 & 1 & 0 & 1 & 2 & 3 & 4 & 5 & 5 & 5 & 4 & 1 & 2 & 3 & 4 & 3 & 2 \\
4 & 3 & 2 & 1 & 0 & 1 & 2 & 3 & 4 & 5 & 6 & 5 & 2 & 2 & 3 & 4 & 4 & 3 \\
5 & 4 & 3 & 2 & 1 & 0 & 1 & 2 & 3 & 4 & 5 & 5 & 2 & 1 & 2 & 3 & 4 & 3 \\
6 & 5 & 4 & 3 & 2 & 1 & 0 & 1 & 2 & 3 & 4 & 5 & 3 & 2 & 2 & 3 & 4 & 4 \\
5 & 5 & 5 & 4 & 3 & 2 & 1 & 0 & 1 & 2 & 3 & 4 & 3 & 2 & 1 & 2 & 3 & 4 \\
4 & 5 & 6 & 5 & 4 & 3 & 2 & 1 & 0 & 1 & 2 & 3 & 4 & 3 & 2 & 2 & 3 & 4 \\
3 & 4 & 5 & 5 & 5 & 4 & 3 & 2 & 1 & 0 & 1 & 2 & 4 & 3 & 2 & 1 & 2 & 3 \\
2 & 3 & 4 & 5 & 6 & 5 & 4 & 3 & 2 & 1 & 0 & 1 & 4 & 4 & 3 & 2 & 2 & 3 \\
1 & 2 & 3 & 4 & 5 & 5 & 5 & 4 & 3 & 2 & 1 & 0 & 3 & 4 & 3 & 2 & 1 & 2 \\
3 & 2 & 2 & 1 & 2 & 2 & 3 & 3 & 4 & 4 & 4 & 3 & 0 & 1 & 2 & 3 & 2 & 1 \\
4 & 3 & 3 & 2 & 2 & 1 & 2 & 2 & 3 & 3 & 4 & 4 & 1 & 0 & 1 & 2 & 3 & 2 \\
4 & 4 & 4 & 3 & 3 & 2 & 2 & 1 & 2 & 2 & 3 & 3 & 2 & 1 & 0 & 1 & 2 & 3 \\
3 & 3 & 4 & 4 & 4 & 3 & 3 & 2 & 2 & 1 & 2 & 2 & 3 & 2 & 1 & 0 & 1 & 2 \\
2 & 2 & 3 & 3 & 4 & 4 & 4 & 3 & 3 & 2 & 2 & 1 & 2 & 3 & 2 & 1 & 0 & 1 \\
2 & 1 & 2 & 2 & 3 & 3 & 4 & 4 & 4 & 3 & 3 & 2 & 1 & 2 & 3 & 2 & 1 & 0
\end{array}\right] .
$$

$W(V)=876$. If $w_{n}$ indicates the Wiener index of $F_{12 n+10}$ then for $n \geq 6$, we have $w_{7}-w_{6}=8640$, $w_{8}-w_{7}=10896, w_{9}-w_{8}=13440, w_{10}-w_{9}=16272$. In general, we get

$$
w_{n}-w_{n-1}=144 n^{2}+96 n+912
$$

and solution of this recurrence is

$$
W\left(F_{12 n+10}\right)=48 n^{3}+120 n^{2}+984 n-1086 .
$$

This completes the proof.

Definition 8. Suppose $x \in V(G)$ is an arbitrary vertex of graph $G$ and $A=A u t(G)$. Then the symmetric total distance $D(x)$ of $x$ can be defined as

$$
D(x)=\sum_{\alpha \in A} d(x, \alpha(x))
$$

We have the following result.

Theorem 9. Let $G$ be a graph with automorphism group $A=A u t(G)$. If $x$ and $y$ are in the same orbit, then $D(x)=D(y)$.

Proof. Suppose $V_{1}, \ldots, V_{k}$ are all orbits of $G$ and $x, y \in V_{i}(1 \leq i \leq k)$ and $V_{i}=\left\{v_{i_{1}}, \ldots, v_{i_{t}}\right\}$. Then the number of automorphisms $\beta$ that map $x$ to $y$, namely $\beta(x)=y$, is $\frac{|A u t(G)|}{\left|V_{i}\right|}$. Hence

$$
D(x)=\sum_{\alpha \in A} d(x, \alpha(x))=\sum_{j=1}^{t} \frac{|A|}{\left|V_{i}\right|} d\left(x, v_{i_{j}}\right)=\sum_{j=1}^{t} \frac{|A|}{\left|V_{i}\right|} d\left(y, v_{i_{j}}\right)=\sum_{\alpha \in A} d(y, \alpha(y))=D(y) .
$$

This completes the proof. 
Example 9. Consider the polyhedral graph $F_{12 n+10}$, for $n=3$ (Figure 21). As we proved in Theorem 5, $\operatorname{Aut}\left(F_{12 n+10}\right)=\mathbb{Z}_{2} \times \mathbb{Z}_{2}$ and we obtain Aut $\left(F_{46}\right)=\mathbb{Z}_{2} \times \mathbb{Z}_{2}$. Also, all orbits of $F_{46}$ are

$$
\begin{array}{lll}
V_{1}=\{1,2,5,6\}, & V_{10}=\{29,35\}, \\
V_{2}=\{3,4\}, & V_{11}=\{30,40,36,34\}, \\
V_{3}=\{7,10,11,14\}, & V_{11}=\{30,40,36,34\}, \\
V_{4}=\{8,9,12,13\}, & V_{12}=\{31,39,37,33\}, \\
V_{5}=\{15,16\}, & V_{13}=\{32,38\}, \\
V_{6}=\{17,24\}, & V_{14}=\{41,44\}, \\
V_{7}=\{18,20,21,23\}, & V_{15}=\{42,43,45,46\} . \\
V_{8}=\{19,22\}, &
\end{array}
$$

For an orbit, for example $V_{1}=\{1,2,5,6\}$, by an easy computation we obtain $D(1)=D(2)=D(5)=$ $D(6)=6$. By using Theorem 9, we have

$$
\begin{aligned}
\widehat{W}\left(F_{46}\right) & =\frac{|V(G)|}{2|A u t(G)|} \sum_{x \in V(x)} \sum_{\alpha \in A} d(x, \alpha(x)) \\
& =\frac{|V(G)|}{2|A u t(G)|} \sum_{x \in V(x)} D(x) \\
& =\frac{|V(G)|}{2|A u t(G)|} \sum_{i=1}^{15}\left|V_{i}\right| \times D\left(x_{i}\right),
\end{aligned}
$$

where $x_{i} \in V_{i}(1 \leq i \leq 15)$ is an arbitrary vertex. On the other hand, $D(3)=1, D(7)=10, D(8)=10$, $D(15)=5, D(17)=6, D(18)=12, D(19)=6, D(25)=12, D(29)=6, D(30)=11, D(31)=12$, $D(32)=5, D(41)=3, D(42)=6$ and using Equation (1) yields that

$$
\widehat{W}\left(F_{46}\right)=\frac{46}{8}(4 \times 6+2 \times 1+4 \times 10+\cdots+4 \times 6)=2185 .
$$

Corollary 9. For the cubic polyhedral graph $F_{12 n+10}(n \geq 3)$ it yields that

$$
\widehat{W}\left(F_{12 n+10}\right)=180 n^{2}+180 n+25 .
$$

Proof. Use Theorem 9 and Example 9 to see that we have

$$
\begin{aligned}
\widehat{W}\left(F_{12 n+10}\right) & =\frac{1}{8}(12 n+10) \sum_{x=1}^{4} \sum_{\alpha \in \mathbb{Z}_{2} \times \mathbb{Z}_{2}} d(x, \alpha(x)) \\
& =\frac{1}{8}(12 n+10)(120 n+20) \\
& =180 n^{2}+180 n+25 .
\end{aligned}
$$

Theorem 10. Suppose $T=T_{z}[10, n]$. For $n \geq 11$, we have

$$
W(T)=\frac{400}{3} n^{3}-800 n^{2}+\frac{24500}{3} n-30700 .
$$


Proof. Let us $U=\left\{u_{1}, \ldots, u_{20}\right\}$ are the vertices of the last layer of $C$ (Figure 4$)$. Hence

$$
\begin{aligned}
2 W(T)=t_{n} & =\sum_{x, y \in U} d(x, y)+\sum_{x, y \in V \backslash U} d(x, y)+2 \sum_{x \in U, y \in V \backslash U} d(x, y) \\
& =2000+t_{n-1}+2 \sum_{x \in U, y \in V \backslash U} d(x, y),
\end{aligned}
$$

and

$$
\sum_{x \in U, y \in V \backslash U} d(x, y)=10\left(d\left(u_{1}\right)+d\left(u_{2}\right)\right),
$$

where $d\left(u_{1}\right)=\sum_{y \in V \backslash U} d\left(u_{1}, y\right)$ and $d\left(u_{2}\right)$ defines similarly, see Figure 24. A direct computation yields that $d\left(u_{1}\right)=20 n^{2}-90 n+330, n \geq 11$, and $d\left(u_{2}\right)=20 n^{2}-110 n+480, n \geq 12$. This implies that $t_{n+1}=2000+t_{n}+10\left(d\left(u_{1}\right)+d\left(u_{2}\right)\right)$ and so

$$
W(T)=\frac{400}{3} n^{3}-800 n^{2}+\frac{24500}{3} n-30700 .
$$

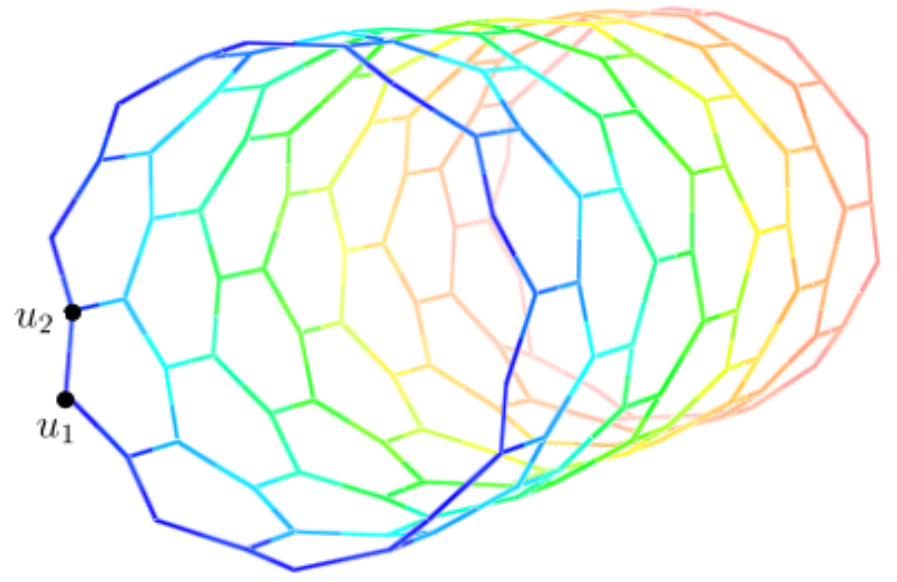

Figure 24. Three-dimensional graph of nanotube $T_{z}[10,7]$.

Theorem 11. For $n \geq 11$,

$$
W\left(F_{20 n+4}\right)=\frac{400}{3} n^{3}+80 n^{2}+\frac{20480}{3} n-20684 .
$$

Proof. By Figure 3, the distance matrix of $F_{20 n+4}$ has the following form

$$
D=\left[\begin{array}{lll}
V & B & W \\
B & U & B \\
W & B & V
\end{array}\right]
$$

where $\left\{v_{1}, v_{2}, \ldots, v_{r}\right\},\left\{u_{1}, \ldots, u_{s}\right\}$ and $\left\{w_{1}, \ldots, w_{r}\right\}$ are the set of vertices of cap $B$, vertices of $T_{z}[10, n]$ and vertices of cap $C$, respectively. The matrix $U$ is the distance matrix of $T_{z}[10, n]$ and $W(V)=6990$. If $w_{n}=W\left(F_{20 n+4}\right)$, then for $n \geq 11$ we have

$$
w_{12}-w_{11}=85,400, w_{13}-w_{12}=101,400, w_{14}-w_{13}=119,000, w_{15}-w_{14}=138,200
$$


Similar to the last cases, we obtain

$$
w_{n}-w_{n-1}=800 n^{2}-4000 n+18200
$$

and consequently

$$
W\left(F_{20 n+4}\right)=\frac{400}{3} n^{3}+80 n^{2}+\frac{20480}{3} n-20684
$$

Corollary 11. For the fullerene graph $F_{20 n+4}$ with $n \geq 3$, we have

$$
\widehat{W}\left(F_{20 n+4}\right)=\left\{\begin{array}{c}
500 n^{2}-350 n-90,2 \mid n, \\
500 n^{2}-260 n-72,2 \not n
\end{array}\right.
$$

Theorem 12. For $n \geq 3$, we obtain

$$
W(N)=288 n^{3}-576 n^{2}+704 n-960,
$$

where $n+1$ is the number of layers of $N=N_{Z}[8, n]$.

Proof. Suppose $U=\left\{u_{1}, u_{2}, \ldots, u_{24}\right\}$ indicates the vertices of the last row. Similar to the last examples, we have

$$
\begin{aligned}
2 W(N)=t_{n} & =\sum_{x, y \in U} d(x, y)+\sum_{x, y \in V \backslash U} d(x, y)+2 \sum_{x \in U, y \in V \backslash U} d(x, y) \\
& =2560+t_{n-1}+2 \sum_{x \in U, y \in V \backslash U} d(x, y),
\end{aligned}
$$

and

$$
\sum_{x \in U, y \in V \backslash U} d(x, y)=8\left(d\left(u_{1}\right)+d\left(u_{2}\right)+d\left(u_{3}\right)\right),
$$

where $d\left(u_{1}\right)=\sum_{y \in V \backslash U} d\left(u_{1}, y\right), d\left(u_{2}\right)$ and $d\left(u_{3}\right)$ defines similarly, see Figure 25 . Then

$$
\begin{aligned}
& d\left(u_{1}\right)=36 n^{2}-60 n-52 \\
& d\left(u_{2}\right)=36 n^{2}-84 n+16 \\
& d\left(u_{3}\right)=36 n^{2}-108 n+72 .
\end{aligned}
$$

This implies that $t_{n+1}=2560+t_{n}+16\left(d\left(u_{1}\right)+d\left(u_{2}\right)+d\left(u_{3}\right)\right)$, and so

$$
W(N)=288 n^{3}-576 n^{2}+704 n-960 .
$$




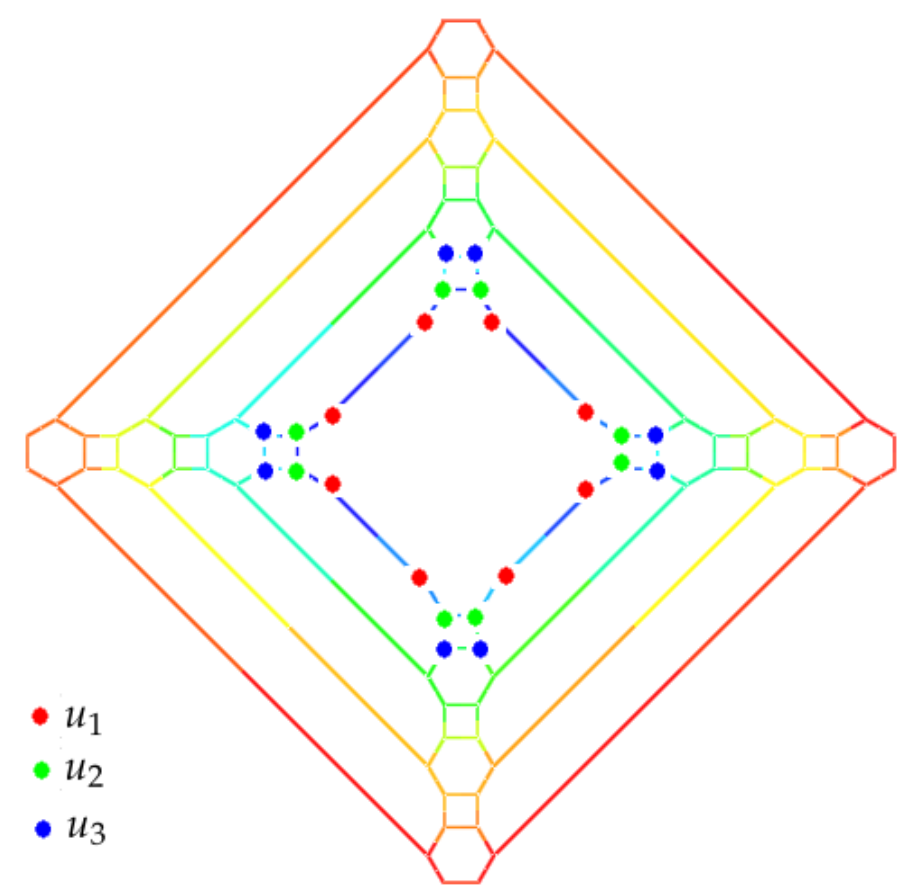

Figure 25. Two-dimensional graph of nanotube $N_{z}[8,3]$.

Theorem 13. For $n \geq 3$, we have

$$
W\left(F_{24 n}\right)=288 n^{3}+576 n^{2}+656 n-956
$$

Proof. Suppose $V\left(F_{24 n}\right)=\left\{v_{1}, v_{2}, \ldots, v_{r}\right\} \cup\left\{u_{1}, u_{2}, \ldots, u_{s}\right\} \cup\left\{w_{1}, w_{2}, \ldots, w_{r}\right\}$, then

$$
D=\left[\begin{array}{lll}
V & B & W \\
B & U & B \\
W & B & V
\end{array}\right]
$$

for $n \geq 3$, we yield that

$$
w_{4}-w_{3}=15344, w_{5}-w_{4}=23408, w_{6}-w_{5}=33200, w_{7}-w_{6}=44720 .
$$

Consequently,

$$
w_{n}-w_{n-1}=864 n^{2}+288 n+368
$$

and thus $W\left(F_{24 n}\right)=288 n^{3}+576 n^{2}+656 n-956$.

Corollary 13. For the cubic polyhedral graph $F_{24 n}$ with $n \geq 3$ we have

$$
\widehat{W}\left(F_{24 n}\right)=1152 n^{2}-636 n \text {. }
$$

Theorem 14. For $n \geq 3$, we have

$$
W(T)=\frac{256}{3} n^{3}+\frac{128}{3} n-256,
$$

where $n+1$ is the number of layers of $T=T_{Z}[8, n]$. 
Proof. Suppose $U=\left\{u_{1}, u_{2}, \ldots, u_{16}\right\}$ are vertices of last row of Figure 26. One can prove that that $t_{n+1}=1024+t_{n}+8\left(d\left(u_{1}\right)+d\left(u_{2}\right)\right)$ and so

$$
W(T)=\frac{256}{3} n^{3}+\frac{128}{3} n-256 .
$$

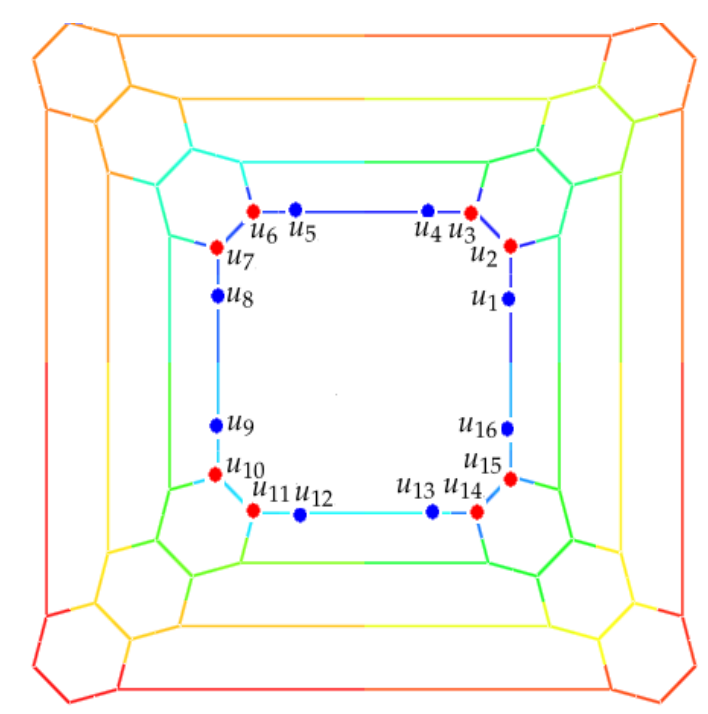

Figure 26. Two-dimensional graph of nanotube $T_{Z}[8,4]$.

Theorem 15. For $n \geq 3$, we obtain

$$
W\left(F_{16 n}\right)=\frac{256}{3} n^{3}+256 n^{2}+\frac{896}{3} n-384 .
$$

Proof. Similar to the lst theorems, suppose

$$
D=\left[\begin{array}{lll}
V & B & W \\
B & U & B \\
W & B & V
\end{array}\right],
$$

Then $W(V)=736$ and so $w_{4}-w_{3}=5248, w_{5}-w_{4}=7808, w_{6}-w_{5}=10880, w_{7}-w_{6}=14464$. This yields to $w_{n}-w_{n-1}=256 n^{2}+256 n+128$ and the solution of this recurrence is

$$
W\left(F_{16 n}\right)=\frac{256}{3} n^{3}+256 n^{2}+\frac{896}{3} n-384 .
$$

Corollary 15. For the cubic polyhedral graph $F_{16 n}(n \geq 3)$, we have

$$
\widehat{W}\left(F_{16 n}\right)=512 n^{2}-320 n .
$$

Theorem 16. For $n \geq 3$,

$$
W\left(F_{24 n+16}\right)=288 n^{3}+1152 n^{2}+1856 n-32 .
$$


Proof. Suppose

$$
D=\left[\begin{array}{lll}
V & B & W \\
B & U & B \\
W & B & V
\end{array}\right]
$$

where $V, B$ and $W$ are distance matrices of cap $B, N_{Z}[8, n]$ and cap $C$ in Figure 17. Also, let $U$ be the distance matrix of $N_{Z}[8, n]$. Similar to the last cases, we have $W(V)=2368$ and for $n \geq 3$, we yield $w_{3}-w_{2}=13088, w_{4}-w_{3}=20576, w_{5}-w_{4}=29792$ and $w_{6}-w_{5}=40736$. This means that

$$
w_{n}-w_{n-1}=864 n^{2}+1440 n+992 .
$$

The solution of this recurrence yields the result.

Corollary 16. For the cubic polyhedral graph $F_{24 n+16}$, we have

$$
\widehat{W}\left(F_{24 n+16}\right)=1152 n^{2}+1056 n+192 .
$$

In Table 1, the Wiener index and the GP-index of five infinite classes of polyhedral graphs, depicted in Figure 27, are reported. Also, in Figures 28 and 29, the behavior of the Wiener and GP-indices of these graphs are depicted. It should be noted that these data are derived from references [19,20,23-25]. Our results show that in the class of polyhedral graphs, two descriptors $W$ and $\widehat{W}$ are highly correlated $\left(R^{2} \approx 0.98\right)$ and capture structural information similarly. Also, we see that the correlation values between the Wiener index and GP-index of trees are also very low, see Table 2. This implies that we can really distinguish these measures on non-polyhedral graphs. Finally, it would be important to perform a similar study to evaluate these measures on different graph classes with more structural diversity.

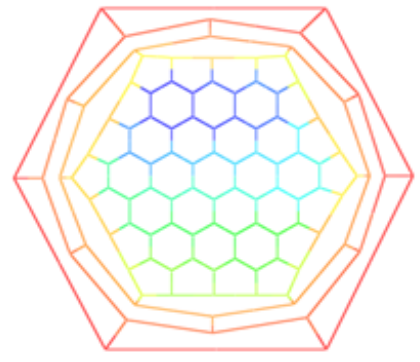

$F_{1}, n \geq 6$

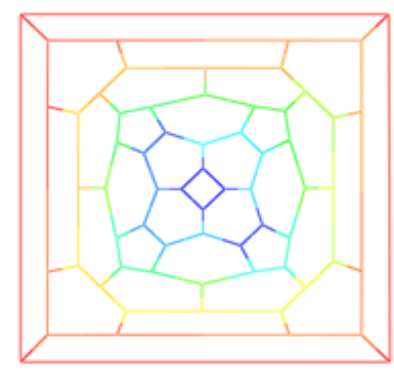

$F_{4}, n \geq 9$

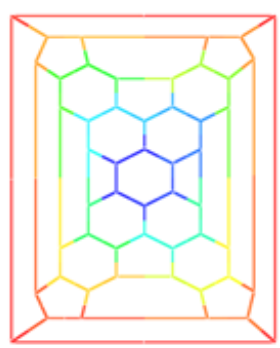

$F_{2}, n \geq 3$

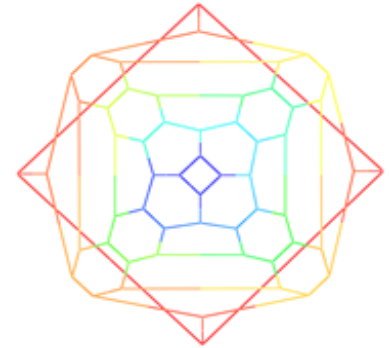

$F_{3}, n \geq 3$

Figure 27. All polyhedral graphs used in Table 1. 
Table 1. Wiener index and Graovac-Pisanski (GP)-index.

\begin{tabular}{ccc}
\hline & $\boldsymbol{W}$ & $\widehat{\boldsymbol{W}}$ \\
\hline$F_{1}$ & $48 n^{3}+862 n^{2}+2988 n+19041$ & $216 n^{2}+3486 n+13140$ \\
$F_{2}$ & $\frac{256}{3} n^{3}+320 n^{2}+\frac{1184}{3} n-474$ & $512 n^{2}-208 n-84$ \\
$F_{3}$ & $\frac{256}{3} n^{3}+384 n^{2}+\frac{1664}{3} n-324$ & $64 n^{3}+480 n^{2}+296 n+36$ \\
$F_{4}$ & $\frac{256}{3} n^{3}+\frac{8384}{3} n-7432$ & $64 n^{3}+256 n^{2}+560 n, n=4 k, k \geq 3$ \\
$64 n^{3}+256 n^{2}+496 n$, otherwise \\
$F_{5}$ & $384 n^{3}+1056 n^{2}+1616 n+36$ & $864 n^{2}+840 n+176$ \\
\hline
\end{tabular}

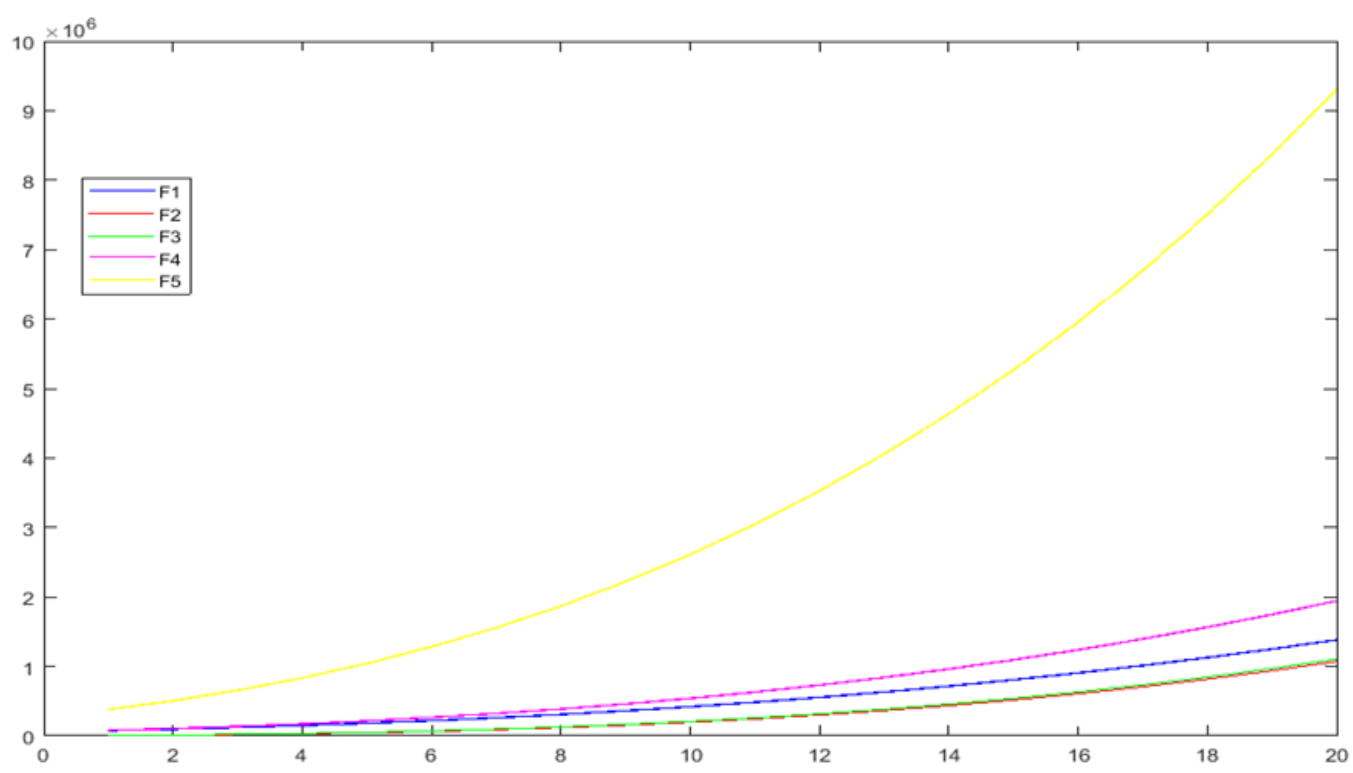

Figure 28. Behavior of the Wiener index of graphs given in Table 1.

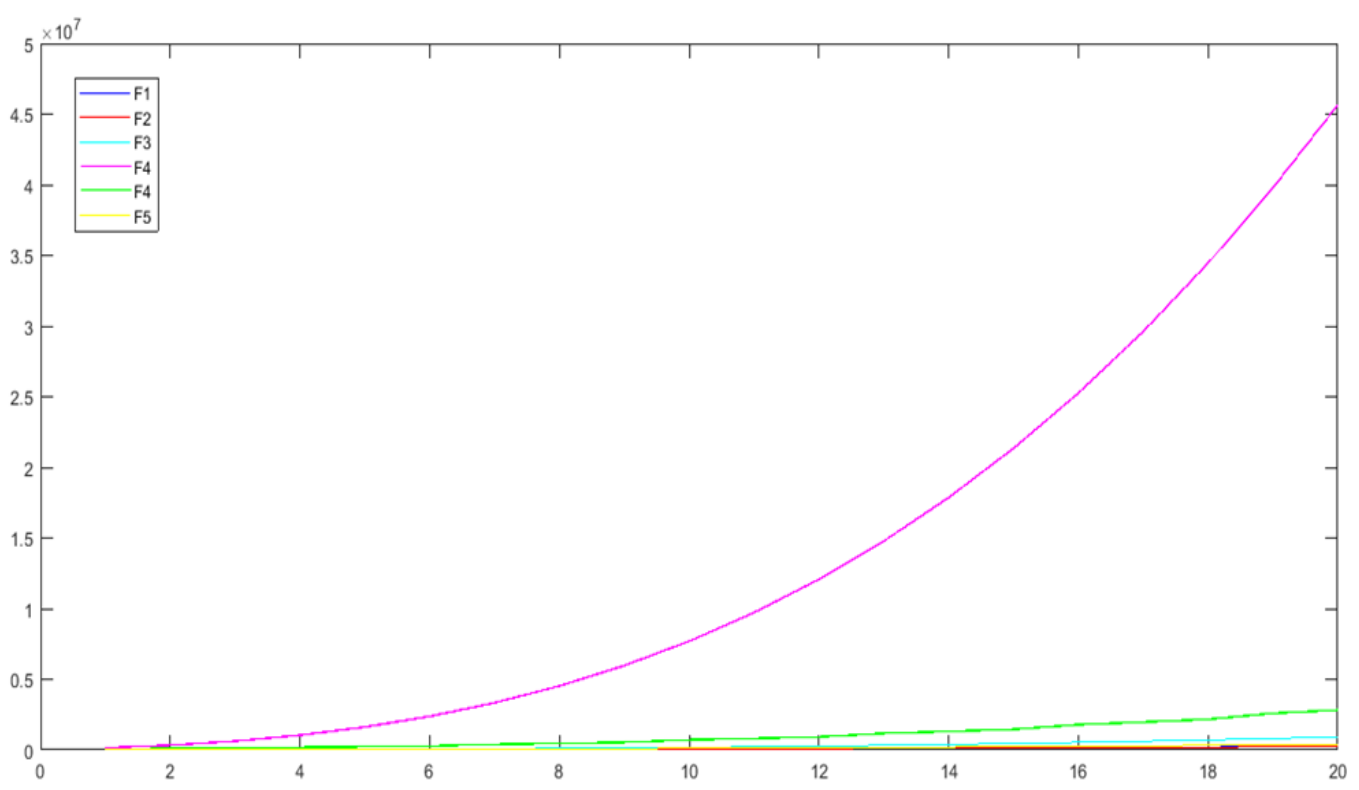

Figure 29. Behavior of the GP-index of graphs given in Table 1.

In Table 2, the correlation between the Wiener index and GP-index of trees of order $5 \leq n \leq 10$ are reported. 
Table 2. The correlation between the Wiener index and GP-index of trees of order $5 \leq n \leq 10$.

\begin{tabular}{cc}
\hline $\boldsymbol{n}$ & Correlation \\
\hline 5 & 0 \\
6 & -0.04 \\
7 & -0.03 \\
8 & -0.06 \\
9 & -0.20 \\
10 & -0.19 \\
\hline
\end{tabular}

\section{Summary and Conclusions}

In this paper, we determined the automorphism group of some classes of cubic polyhedral graphs and then we investigated their Wiener and Graovac-Pisanski indices. An analysis of all data shows that the correlation values between the Wiener and GP-indices is very high and meaningful but these graph descriptors have quite a low correlation. Therefore, this implies that we cannot really distinguish these measures on cubic polyhedral graphs.

Author Contributions: M.G., M.H.-N., M.D., and X.L. wrote the paper. All authors have read and agreed to the published version of the manuscript.

Funding: Matthias Dehmer thanks the Austrian Science Funds for supporting this work (P30031).

Conflicts of Interest: The authors declare no conflict of interest.

\section{References}

1. Wiener, H.J. Structural Determination of Paraffin Boiling Points. J. Am. Chem. Soc. 1947, 69, 17-20. [CrossRef] [PubMed]

2. Harary, F. Graph Theory; Addison-Wesley: Reading, MA, USA, 1969.

3. Dobrynin, A.A.; Entringer, R.; Gutman, I. Wiener index of trees: Theory and applications. Acta Appl. Math. 2001, 66, 211-249. [CrossRef]

4. Guo, H.; Zhou, B.; Lin, H. The Wiener index of uniform hypergraphs. MATCH Commun. Math. Comput. Chem. 2017, 78, 133-152.

5. Knor, M.; Škrekovski, R. Wiener index of line graphs. In Quantitative Graph Theory: Mathematical Foundations and Applications; Dehmer, M., Emmert-Streib, F., Eds.; CRC Press: Boca Raton, FL, USA, 2014; pp. $279-301$.

6. Knor, M.; Škrekovski, R.; Tepeh, A. Mathematical aspects of wiener index. Ars Math. Contemp. 2016, 11,327-352. [CrossRef]

7. Xu, K.; Liu, M.; Das, K.C.; Gutman, I.; Furtula, B. A survey on graphs extremal with respect to distance-based topolgical indices. MATCH Commun. Math. Comput. Chem. 2014, 71, 461-508.

8. Graovac, A.; Pisanski, T. On the Wiener index of a graph. J. Math. Chem. 1991, 8, 53-62. [CrossRef]

9. Črepnjak, M.; Tratnik, N.; Pleteršek, P.Ž. Predicting melting points of hydrocarbons by the Graovac-Pisanski index. Fuller. Nanotub. Carbon Nanostruct. 2016, 26, 239-245. [CrossRef]

10. Gutman, I.; Šoltés, L. The range of the Wiener index and its mean isomer degeneracy. Z. Naturforschung 1991, 46, 865-868. [CrossRef]

11. Ashrafi, A.R.; Shabani, H. The modified Wiener index of some graph operations. Ars Math. Contemp. 2016, 11, 277-284.

12. Koorepazan-Moftakhar, F.; Ashrafi, A.R. Distance under symmetry. MATCH Commun. Math. Comput. Chem. 2015, 74, 259-272.

13. Ghorbani, M.; Klavžar, S. Modified Wiener index via canonical metric representation, and some fullerene patches. Ars Math. Contemp. 2016, 11, 247-254. [CrossRef]

14. Tratnik, N. The Graovac-Pisanski index of zig-zag tubulenes and the generalized cut method. J. Math. Chem. 2017, 55, 1622-1637. [CrossRef]

15. Knor, M.; Škrekovski, R.; Tepeh, A. Trees with the maximum value of Graovac- Pisanski index. Appl. Math. Comput. 2019, 358, 287-292. 
16. Knor, M.; Komorník, J.; Škrekovski, R.; Tepeh, A. Unicyclic graphs with the maximal value of Graovac-Pisanski index. Ars Math. Contemp. 2019, 17, 455-466. [CrossRef]

17. Knor, M.; Škrekovski, R.; Tepeh, A. On the Graovac-Pisanski index of a graph. Acta Math. Univ. Comen. 2019, 88, 867-870.

18. Črepnjak, M.; Knor, M.; Tratnika, N.; Pleteršek, P.Ž. The Graovac-Pisanski index of a connected bipartite graph is an integer number. arXiv 2017, arXiv:1709.04189v1.

19. Hakimi-Nezhaad, M.; Ghorbani, M. Differences between Wiener and modified Wiener indices. J. Math. NanoSci. 2014, 4, 19-25.

20. Ghorbani, M.; Hakimi-Nezhaad, M. An algebraic study of non-classical fullerenes. Fuller. Nanotub. Carbon Nanostruct. 2016, 24, 385-390. [CrossRef]

21. Knor, M.; Škrekovski, R.; Tepeh, A. On the difference between Wiener index and Graovac-Pisanski index. MATCH Commun. Math. Comput. Chem. 2020, 83, 109-120.

22. Ghorbani, M. Fullerene graphs with pentagons and heptagons. J. Math. Nanosci. 2013, 3, 33-37.

23. Ghorbani, M.; Hakimi-Nezhaad, M.; Abbasi Barfaraz, F. An algebraic approach to Wiener number. J. Appl. Math. Comput. 2016, 55, 629-643. [CrossRef]

24. Ghorbani, M.; Hakimi-Nezhaad, M. Polyhedral graphs under automorphism groups. Stud. Ubb Chem. 2016, LXI, 261-272.

25. Hakimi-Nezhaad, M.; Ghorbani, M. On the Graovac-Pisanski index. Kragujev. J. Sci. 2017, 39, 91-98. [CrossRef]

26. Ghorbani, M.; Hakimi-Nezhaad, M. Study of fullerenes via their symmetry groups. Fuller. Nanotub. Carbon Nanostruct. 2017, 25, 613-623. [CrossRef]

27. Khaksari, A.; Hakimi-Nezhaad, M.; Ori, O.; Ghorbani, M. A survey of the automorphism groups of some fulleroids. Fuller. Nanotub. Carbon Nanostruct. 2018, 26, 80-86. [CrossRef]

28. Koorepazan-Moftakhar, F.; Ashrafi, A.R.; Ori, O. Symmetry groups and Graovac-Pisanski index of some linear polymers. Quasigroups Relat. Sys. 2018, 26, 87-102.

29. Ashrafi, A.R.; Koorepazan-Moftakhar, F.; Diudea, V.M.; Ori, O. Graovac-Pisanski index of fullerenes and fullerene-like molecules. Fuller. Nanotub. Carbon Nanostruct. 2016, 24, 779-785. [CrossRef]

30. Madani, S.; Ashrafi, A.R. Symmetry and two symmetry measures for the web and spider web graphs. J. Appl. Math. Comput. 2020, 1-12. [CrossRef]

31. Tratnik, N.; Pleteršek, P.Ž. The Graovac-Pisanski index of armchair nanotubes. J. Math. Chem. 2018, 56, 1103-1116. [CrossRef]

32. Dixon, J.D.; Mortimer, B. Permutation Groups; Springer: Berlin/Heidelberg, Germany, 1966.

33. Deza, M.; Dutour Sikirić, M.; Fowler, P.W. Zigzags, railroads, and knots in fullerenes. J. Chem. Inf. Comp. Sci. 2004, 44, 1282-1293. [CrossRef]

34. Dutour Sikirić, M.; Delgado-Friedrichs, O.; Deza, M. Space fullerenes: Computer search for new Frank-Kasper structures. Acta Crystallogr. A 2010, 66, 602-615. [CrossRef] [PubMed]

35. Fowler, P.W.; Manolopoulos, D.E. An Atlas of Fullerenes; Oxford University Press: New York, NY, USA, 1995.

36. Zhang, H.; Ye, D. An upper bound for the Clar number of fullerene graphs. J. Math. Chem. 2007, 41, 123-133. [CrossRef]

37. Ghorbani, M.; Dehmer, M.; Emmert-Streib, F. Properties of entropy-based topological measures of fullerenes. Mathematics 2020, 8, 740. [CrossRef]

38. Ghorbani, M.; Dehmer, M.; Mowshowitz, A.; Tao, J.; Emmert-Streib, F. The Hosoya entropy of graphs revisited. Symmetry 2019, 11, 1013. [CrossRef]

39. Ghorbani, M.; Dehmer, M.; Rahmani, S.; Rajabi-Parsa, M. A Survey on symmetry group of polyhedral graphs. Symmetry 2020, 12, 370. [CrossRef]

(C) 2020 by the authors. Licensee MDPI, Basel, Switzerland. This article is an open access article distributed under the terms and conditions of the Creative Commons Attribution (CC BY) license (http:/ / creativecommons.org/licenses/by/4.0/). 